\title{
Convective heat transfer in a dusty ferromagnetic fluid over a stretching surface with prescribed surface temperature/heat flux including heat source/sink
}

\author{
A. Majeed ${ }^{1}$, A. Zeeshan ${ }^{2 *}$ and R.S.R. Gorla ${ }^{3}$ \\ ${ }^{1}$ Department of Mathematics, Bacha Khan University, Charsadda, KPK, Pakistan. \\ ${ }^{2}$ Department of Mathematics and Statistics, Faculty of Basic and Applied Sciences, International Islamic University, Islamabad, 44000, Pakistan. \\ ${ }^{3}$ Department of Mechanical Engineering, Cleveland State University, Cleveland, OH 44115, USA.
}

\begin{abstract}
The aim of the present paper was to investigate the boundary layer flow and heat transfer in a dusty fluid with ferromagnetic particles over a flat stretching sheet including the effect of magnetic dipole. By using suitable similarity transformations, the governing partial differential equations of momentum and energy were reduced into non-linear ordinary differential equations. The resulting differential equations with corresponding boundary conditions were solved numerically by employing shooting based fourth order Runge-Kutta method. The effect of controlling parameters such as ferromagnetic interaction parameter, mixed convection parameter, Eckert number, Prandtl number, the number of dust particles and heat generation/absorption parameter on the temperature and velocity profiles are considered graphically for two types of heating processes, namely, the prescribed surface temperature (PST) and the prescribed heat flux (PHF). The value of skin friction coefficient and Nusselt number are presented in tabular form for different values of the governing parameters. It is found that the value of local Nusselt number increases with an increase of the value of heat source/sink parameter for both PST and PHF cases. The present results were compared with previously published data for a special case and an excellent agreement was found.
\end{abstract}

Keywords: Dusty fluid, ferro-particles, heat source/sink, magnetic dipole, mixed convection, stretching sheet.

\section{INTRODUCTION}

Ferrofluids are made from magnetic nano-particles suspended uniformly in base liquid and has an average size of 5 - $10 \mathrm{~nm}$ (Rosensweig et al., 1965). Ferrofluid flow and energy transport can be controlled by using an external magnetic field. These fluids have attracted the attention of many scientists and researchers because of their numerous applications in engineering, including microfluidic actuators, lithographic patterning, microelectro mechanical system (MEMS), seal technology, optical and sensor applications, and lubrication of bearing and dumpers. Ferrofluids can also be used in treating cancer by heating the tumor soaked in ferrofluids by means of an alternating magnetic field (Feynman et al., 1963; Ganguly et al., 2004; Shliomis, 2004; Song et al., 2006; Strek \& Jopek, 2007; Chang et al., 2009). The impact of non-uniform magnetic field on forced convective heat transfer of ferrofluid by applying the finite element method has been analysed (Sheikholeslami et al., 2015). The study showed that the Nusselt number is directly proportional to the Reynolds number and nano-particle volume fraction, although it is reversely associated with the Hartmann number. Effect of applied magnetic field on the flow of heated ferrofluid in a semi annulus enclosure using the control volume finite element method (CVFEM) was illustrated by Sheikholeslami and Ganji (2014). Heat transfer analysis of water-based $\mathrm{Fe}_{3} \mathrm{O}_{4}$ ferrofluid through a mini channel in the presence of constant and alternating magnetic fields was discussed by Ghasemian et al. (2015). Free convection of $\mathrm{Fe}_{3} \mathrm{O}_{4}-$ water-based nanofluid with ferrohydrodynamic (FHD) and magnetohydrodynamic (MHD) has been proposed

\footnotetext{
* Corresponding author (ahmad.zeeshan@iiu.edu.pk; (D) https://orcid.org/0000-0002-2641-1575)
} 
by Sheikholeslami and Rashidi (2015). The thermal behaviour of ferrofluid in a vertical pipe in the presence of a non-uniform magnetic field was studied (Aminfar et al., 2011). The study used a two phase model and established that there is an increase in heat transfer coefficient when the magnetic field gradient is negative and velocity distribution becomes more uniform. The study of nanofluid flow past a nonlinear isothermal stretching sheet in the presence of porous medium has been examined analytically by Rashidi et al. (2014a). The natural convection of ferrofluid flow in a linearly heated cavity was performed by Kefayati (2014), and showed that heat transfer decreases as the volume fraction of ferromagnetic particle increases.

Heat transfer analysis in dusty fluid flow over a flat stretching surface has gained much interest due to its numerous science and engineering applications such as metallurgical process and polymer extrusion process. The governing equations for dusty fluid has been modelled (Saffman, 1962) and the laminar flow of dusty gas was analysed, in which particles are distributed uniformly. More recently, the heat transfer effect on dusty fluid over a stretching sheet with the influence of non-uniform heat source/sink has been investigated (Gireesha et al., 2011). MHD boundary layer flow and heat transfer of nanofluid embedded with homogenous dust particles have also been discussed (Gireesha et al., 2015). Heat transfer in water-based nanofluid over a vertical stretching sheet with MHD impact was investigated (Rashidi et al., 2014b). Suspended particle effect on viscous nanofluid in the presence of porous medium past a stretching sheet was studied by Gireesha et al. (2014), where they considered four different classes of nanoparticles: copper $(\mathrm{Cu})$, copper oxide $(\mathrm{CuO})$, silver $(\mathrm{Ag})$ and alumina $\left(\mathrm{Al}_{2} \mathrm{O}_{3}\right)$. Unsteady laminar free convective nanofluid flow in the presence of a transverse magnetic field has been reviewed (Freidoonimehr et al., 2015). It was found that by decreasing the nano-particle volume fraction, there is a decrease in the skin friction coefficient. The steady MHD flow of viscous fluid between radially stretching sheets filled with a porous medium under the influence of Dufour and Soret effects was investigated by Nawaz et al. (2012). The influence of Brownian motion and thermophoresis on the axisymmetric boundary layer flow of nanofluid induced by radial stretching sheet was also considered (Nawaz \& Hayat, 2014). The twodimensional boundary layer flow of tangent hyperbolic fluid towards a stretching surface with magnetic field effects was discussed (Akbar et al., 2013). Some recent studies associated with heat transfer and ferromagnetic fluid were carried out by Majeed et al. (2016a; 2016b), Rehman et al. (2017), Zeeshan et al. (2016;
2017), Zeeshan and Majeed (2016), and Shehzad et al. (2016).

The purpose of the present study was to explore the heat transfer analysis of dusty fluid with magnetic particles past a stretching surface in the presence of dipole effect. In addition, the effect of several physical parameters on dimensionless velocity and temperature profile was analysed for two types of boundary conditions: prescribed surface temperature (PST) and prescribed heat flux (PHF).

\section{MATHEMATICAL FORMULATION}

\section{Flow analysis}

Consider the two-dimensional steady incompressible boundary layer flow and heat transfer of viscous dusty fluid with ferromagnetic particles past a stretching surface under the effect of applied magnetic field generated by magnetic dipole. A stretching sheet is considered along the $x$-axis with velocity $u_{w}=c x$ and the $y$-axis is normal to the surface as shown in Figure 1. A magnetic dipole is situated on the centre of the $y$ axis at distance ' $a$ ' from the sheet, whose magnetic field points in the positive $\mathrm{x}$ direction and gives rise to a magnetic field of sufficient strength to saturate the ferrofluid. It is also supposed that the uniform temperature at the surface of the sheet is $T_{w}(x, t)$ and Curie temperature $T_{c}$, while the temperature of the ambient ferrofluid far from the surface of the sheet is $T_{\infty}=T_{c}$ and unable to magnetise until they start to cool after entering into the thermal boundary layer area near the sheet.

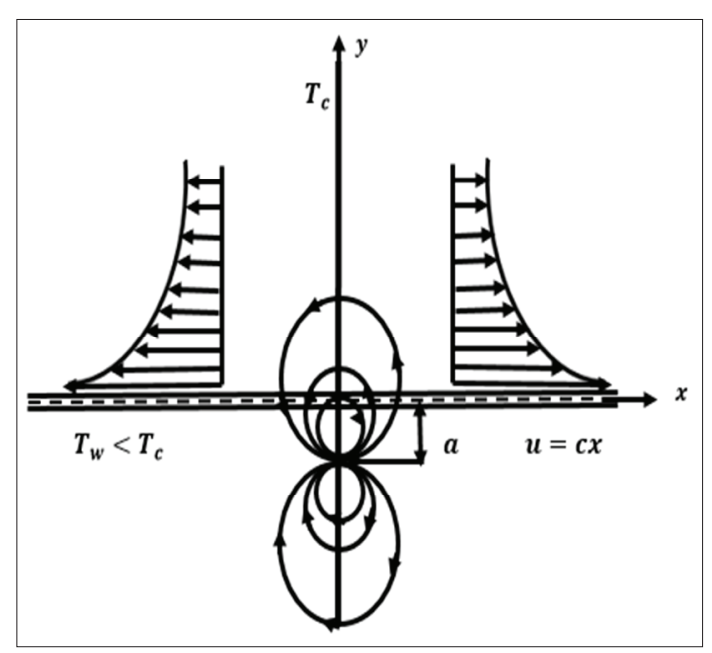

Figure 1: Geometry of the problem 


\section{Magnetic dipole}

The magnetic scalar potential of ferrofluid affected by the external magnetic field due to dipole effect is given as (Andersson \& Valnes, 1998):

$\Phi=\frac{\gamma}{2 \pi}\left(\frac{x}{x^{2}+(y+a)^{2}}\right)$,

where $\gamma$ is the magnetic field strength at the source. Components of magnetic field intensity, $H_{x}$ and $H_{y}$ along the $x$ and $y$ directions are given below.

$$
\begin{aligned}
& H_{x}=-\frac{\partial \Phi}{\partial x}=\frac{\gamma}{2 \pi}\left\{\left(\frac{x^{2}-(y+a)^{2}}{\left(x^{2}+(y+a)^{2}\right)^{2}}\right)\right\}, \\
& H_{y}=-\frac{\partial \Phi}{\partial y}=\frac{\gamma}{2 \pi}\left\{\left(\frac{2 x(y+a)}{\left(x^{2}+(y+a)^{2}\right)^{2}}\right)\right\} .
\end{aligned}
$$

The magnetic force is equivalent to the gradient of the magnitude of $H$. After having expanded in powers of $x$ and retained terms up to order $x^{2}$, we get,

$$
\begin{aligned}
& H=\sqrt{\left(\frac{\partial \Phi}{\partial x}\right)^{2}+\left(\frac{\partial \Phi}{\partial y}\right)^{2}}, \\
& \frac{\partial H}{\partial x}=-\frac{\gamma}{2 \pi}\left(\frac{2 x}{(y+a)^{4}}\right), \\
& \frac{\partial H}{\partial y}=\frac{\gamma}{2 \pi}\left(\frac{-2}{(y+a)^{3}}+\frac{4 x^{2}}{(y+a)^{5}}\right) .
\end{aligned}
$$

The variation of magnetisation $M$ can be considered as a linear function of temperature as assumed by Andersson and Valnes (1998)

$$
M=K^{*}\left(T_{c}-T\right)
$$

where $K^{*}$ is a pyromagnetic coefficient and $T_{c}$ is the Curie temperature. However, the following points are essential for the occurrence of ferrohydrodynamic: (i) fluid is at a temperature $T$ different from $T_{c}$ and (ii) the external magnetic field is inhomogeneous. Once the ferrofluid approaches to Curie temperature, there is no further magnetisation. Characteristics for physical point of view is very important, because the Curie temperature is very high; $1,043 \mathrm{~K}$ for iron.

\section{Dusty ferrofluid phase}

Using the boundary layer approximation, twodimensional boundary layer equations of mass and momentum for dusty ferrofluid are (Nandkeolyar et al., 2013):

$\frac{\partial u}{\partial x}+\frac{\partial v}{\partial y}=0$

$\left(u \frac{\partial u}{\partial x}+v \frac{\partial u}{\partial y}\right)=\frac{\mu_{0}}{\rho} M \frac{\partial H}{\partial x}+\frac{\mu}{\rho}\left(\frac{\partial^{2} u}{\partial x^{2}}+\frac{\partial^{2} u}{\partial y^{2}}\right)+\frac{K_{1} N}{\rho}\left(u_{p}-u\right)$

$+g \beta^{*}\left(T_{c}-T\right)$

$\left(u \frac{\partial v}{\partial x}+v \frac{\partial v}{\partial y}\right)=\frac{\mu_{0}}{\rho} M \frac{\partial H}{\partial y}+\frac{\mu}{\rho}\left(\frac{\partial^{2} v}{\partial x^{2}}+\frac{\partial^{2} v}{\partial y^{2}}\right)+\frac{K_{1} N}{\rho}\left(v_{p}-v\right)$

$+g \beta^{*}\left(T_{c}-T\right)$

$u_{p} \frac{\partial u_{p}}{\partial x}+v_{p} \frac{\partial u_{p}}{\partial y}=\frac{K_{1}}{m}\left(u-u_{p}\right)$

$u_{p} \frac{\partial v_{p}}{\partial x}+v_{p} \frac{\partial v_{p}}{\partial y}=\frac{K_{1}}{m}\left(v-v_{p}\right)$

$\frac{\partial\left(\rho_{p} u_{p}\right)}{\partial x}+\frac{\partial\left(\rho_{p} v_{p}\right)}{\partial y}=0$

where $(u, v)$ and $\left(u_{p}, v_{p}\right)$ represent the velocity components of ferrofluid and dust particles along the $x$ and $y$ axis, respectively. $\rho$ and $\rho_{p}$ are densities of the ferrofluid and dust phase, respectively, $K_{1}$ is the Stoke's resistance coefficient, $\mu$ is the dynamic viscosity, $v=\frac{\mu}{\rho}$ is the kinematic viscosity, $\mu_{0}$ is the magnetic permeability, $g$ is the acceleration due to gravity, $\beta^{*}$ is the volumetric coefficient of thermal expansion, $m$ and $N$ are the mass concentration and the number density of the particle phase, respectively, $M$ is the magnetisation and $H$ is the magnetic field. The terms $\mu_{0} M \frac{\partial H}{\partial x}$ and $\mu_{0} M \frac{\partial H}{\partial y}$ in equations (9) and (10) indicate the components of the magnetic body force per unit volume and rely on the existence of the magnetic gradient. These forces will be disappeared in the absence of magnetic gradient. 
The corresponding boundary conditions related to the above problem are:

$\left.\begin{array}{l}u=u_{w}(x)=c x, \quad v=0, \quad \text { at } y=0 \\ u \rightarrow 0, \quad u_{p} \rightarrow 0, \quad v_{p} \rightarrow 0 \quad \rho_{p} \rightarrow E \rho \text { as } y \rightarrow \infty\end{array}\right\}$,

where $c>0$ is the stretching parameter of sheet and $E$ is the density ratio. To convert the governing equations in dimensionless form, we consider the following similarity transformation

$$
\begin{aligned}
& \Psi(\xi, \eta)=\left(\frac{\mu}{\rho}\right) \xi . f(\eta), \\
& \xi=\sqrt{\frac{c \rho}{\mu}} x, \quad \eta=\sqrt{\frac{c \rho}{\mu}} y .
\end{aligned}
$$

The velocity components are defined as

$$
\begin{aligned}
& u=\frac{\partial \Psi}{\partial y}=c x \cdot f^{\prime}(\eta), \quad v=-\frac{\partial \Psi}{\partial x}=-\sqrt{c v} \cdot f(\eta), \\
& u_{p}=c x F(\eta), \quad v_{p}=\sqrt{c v} \cdot G(\eta), \rho_{r}=\frac{\rho_{p}}{\rho}=W(\eta),
\end{aligned}
$$

where $\rho_{r}$ is the relative density. Substituting equations (14) - (17) into the equations (9) - (13) and comparing the coefficients of, such as powers of $\xi$ up to order $\xi^{2}$, we get the following non-linear differential equations.

$$
\begin{aligned}
& f^{\prime \prime \prime}+f f^{\prime \prime}-f^{\prime 2}-\frac{2 \beta \theta_{1}}{\left(\eta+\alpha_{1}\right)^{4}}+l_{1} \alpha W(\eta)\left(F-f^{\prime}\right)+G^{*} \theta_{1}=0, \\
& f^{\prime \prime}+f f^{\prime}+\frac{2 \beta \theta_{1}}{\left(\eta+\alpha_{1}\right)^{3}}-l_{1} \alpha W(\eta)(G+f)+G^{*} \theta_{1}=0, \\
& F^{2}+F^{\prime} G-\alpha\left(f^{\prime}-F\right)=0, \\
& G G^{\prime}+\alpha(f+G)=0, \\
& F W+W^{\prime} G+W G^{\prime}=0,
\end{aligned}
$$

where $\beta=\frac{\gamma \rho}{2 \pi \mu^{2}} \mu_{0} K^{*}\left(T_{c}-T_{w}\right)$ is the ferromagnetic interaction parameter, $l_{1}=\frac{m N}{\rho_{p}}$ is the mass concentration of dust particles, $\alpha=\frac{1}{\tau c}$ is the fluid particle interaction parameter, $G^{*}=\frac{G r_{x}}{\operatorname{Re}_{x}^{2}}$, is the mixed convection parameter, $G r_{x}=\frac{g \beta^{*}\left(T_{c}-T_{w}\right)}{v^{2}}$ is the local Grashof number, and $\alpha_{1}=\sqrt{\frac{c \rho}{\mu}} a$ is the dimensionless distance.

Using the transformation (15) - (17) in the boundary conditions equation (14) takes the form:

$$
\left.\begin{array}{l}
f=0, f^{\prime}=1, \text { at } \eta=0 \\
f^{\prime} \rightarrow 0, F \rightarrow 0, G \rightarrow-f, W \rightarrow E \text { as } \eta \rightarrow \infty
\end{array}\right\},
$$

\section{Heat transfer analysis}

Heat transport equations for dusty ferrofluid for twodimensional flow in the presence of heat generation/ absorption are as follows (Nandkeolyar et al., 2013):

$$
\begin{aligned}
& \left(u \frac{\partial T}{\partial x}+v \frac{\partial T}{\partial y}\right)+\frac{\mu_{0}}{\rho c_{p}} T \frac{\partial M}{\partial T}\left(u \frac{\partial H}{\partial x}+v \frac{\partial H}{\partial y}\right)=\frac{k}{\rho c_{p}} \frac{\partial^{2} T}{\partial y^{2}}+ \\
& \frac{\mu}{\rho c_{p}}\left(\frac{\partial u}{\partial y}\right)^{2}+\frac{N c_{p}}{\rho c_{p} \tau_{T}}\left(T_{p}-T\right)+\frac{N}{\rho c_{p} \tau_{v}}\left(u_{p}-u\right)+\frac{Q_{0}}{\rho c_{p}}\left(T_{c}-T\right),
\end{aligned}
$$

$u_{p} \frac{\partial T_{p}}{\partial x}+v_{p} \frac{\partial T_{p}}{\partial y}=\frac{c_{p}}{c_{m} \tau_{T}}\left(T_{p}-T\right)$,

where $T$ and $T_{p}$ are the temperature of ferrofluid and dust particle inside the boundary layer, respectively, $Q_{0}$ is the heat generation/absorption coefficient, the second term in the left-hand side of equation (24) represent the heating due to adiabatic magnetisation. $\tau_{v}=m / K_{1}$ is the relaxation time of dust particle, $k$ is the thermal conductivity and $\tau_{T}$ is the thermal equilibrium time. $c_{p}$ and $c_{m}$ are the specific heat of ferromagnetic and dust particles, respectively.

The heat transfer analysis has been performed for two different types of boundary conditions:

$\left.\begin{array}{l}T=T_{w}=T_{c}-A\left(\frac{x}{l}\right)^{2} \quad \text { for PST } \\ -k \frac{\partial T}{\partial y}=q_{w}=D\left(\frac{x}{l}\right)^{2} \text { for PHF }\end{array}\right\}$ at $y=0$,

$T \rightarrow T_{c}, \quad T_{p} \rightarrow T_{c} \quad$ as $y \rightarrow \infty$ 
where $A$ and $D$ are positive constants and $l=\sqrt{\frac{v}{c}}$ is the characteristic length. Introducing the dimensionless variable for ferrofluid phase $\theta(\eta)$ and dust phase $\theta_{p}(\eta)$ temperature:

$$
\theta(\xi, \eta) \equiv \frac{T_{c}-T}{T_{c-} T_{w}}=\theta_{1}(\eta)+\xi^{2} \theta_{2}(\eta), \theta_{p}(\eta)=\frac{T_{c}-T_{p}}{T_{c-} T_{w}},
$$

where

$$
\left.\begin{array}{ll}
T_{c-} T_{w}=A\left(\frac{x}{l}\right)^{2} & \text { for PST case } \\
T_{c-} T_{w}=\frac{D}{k}\left(\frac{x}{l}\right)^{2} \sqrt{\frac{v}{c}} & \text { for PHF case }
\end{array}\right\} .
$$

Using the similarity variable stated in equations (15) and (27) into equations (24) and (25) and equating coefficients up to $\xi^{2}$ on both sides, we get the following system of equations:

$$
\begin{aligned}
& \theta_{1}^{\prime \prime}+\operatorname{Pr}\left(E c f^{\prime \prime 2}+c_{1} N\left(\theta_{p}-\theta_{1}\right)+c_{2} N\left(F-f^{\prime}\right)^{2}-2 f^{\prime} \theta_{1}+\right. \\
& \left.f \theta_{1}^{\prime}+H_{1} \theta_{1}\right)+\frac{2 \lambda \beta\left(\theta_{1}-\varepsilon\right) f}{\left(\eta+\alpha_{1}\right)^{3}}=0 \\
& \theta_{2}^{\prime \prime}+\operatorname{Pr}\left(-c_{1} N \theta_{2}-4 f^{\prime} \theta_{2}+f \theta_{2}^{\prime}+H_{1} \theta_{2}\right)-\lambda \beta\left(\theta_{1}-\varepsilon\right) \\
& {\left[\frac{2 f^{\prime}}{\left(\eta+\alpha_{1}\right)^{4}}+\frac{4 f}{\left(\eta+\alpha_{1}\right)^{5}}\right]+\frac{2 \lambda \beta f \theta_{2}}{\left(\eta+\alpha_{1}\right)^{3}}=0} \\
& G \theta_{p}^{\prime}+2 F \theta_{p}+c_{3}\left(\theta_{p}-\theta_{1}\right)=0
\end{aligned}
$$

where $\operatorname{Pr}=\frac{\mu c_{p}}{k}$ is the Prandtl number, $H_{1}=\frac{Q_{\circ}}{c \rho c_{p}}$ is the heat source $\left(H_{1}>0\right)$ or sink $\left(H_{1}<0\right), \lambda=\frac{c \mu^{2}}{\rho k\left(T_{c}-T_{w}\right)}$ is the viscous dissipation parameter, $E c=\frac{U_{w}^{2}}{c_{p}\left(T_{c}-T\right)}$ is the Eckert number, $\varepsilon=\frac{T_{c}}{T_{c}-T_{w}}$ is the dimensionless Curie temperature ratio, $c_{1}=\frac{1}{\rho c \tau_{T}}$ and $c_{3}=\frac{c_{p}}{c_{m} c \tau_{T}}$ are the fluid particle interaction parameters for heat transfer, $c_{2}=\frac{1}{\rho c \tau_{v}}$ is the fluid particle interaction parameter for velocity.
The thermal boundary conditions (26) are transformed as:

$$
\left.\begin{array}{l}
\theta_{1}=1, \quad \theta_{2}=0, \quad \text { for PST Case } \\
\theta_{1}^{\prime}=-1, \quad \theta_{2}^{\prime}=0, \quad \text { for PHF Case }
\end{array}\right\} \text { at } \eta=0,
$$

The important physical parameters for practical interest, the local skin friction coefficient and the local Nusselt number can be expressed as:

$$
C_{f_{x}}=\frac{-2 \tau_{w}}{\rho(c x)^{2}}, \quad N u_{x}=\frac{x q_{w}}{-k\left(T_{c}-T\right)},
$$

where $\tau_{w}$ is the shear stress and $q_{w}$ is the heat flux given by

$$
\tau_{w}=\mu\left(\frac{\partial u}{\partial y}\right)_{y=0}, q_{w}=-k\left(\frac{\partial T}{\partial y}\right)_{y=0}
$$

Using the similarity variables, we get

$$
\left.\begin{array}{ll}
\operatorname{Re}_{x}^{1 / 2} C_{f}=-2 f^{\prime \prime}(0) & \\
N u_{x} / \operatorname{Re}_{x}^{1 / 2}=-\left(\theta_{1}^{\prime}(0)+\xi^{2} \theta_{2}^{\prime}(0)\right) & \mathrm{PST} \\
N u_{x} / \operatorname{Re}_{x}^{1 / 2}=1 /\left(\theta_{1}(0)+\xi^{2} \theta_{2}(0)\right) & \mathrm{PHF}
\end{array}\right\},
$$

where $f^{\prime \prime}(0)$ is the dimensionless skin friction coefficient, $\theta_{1}^{\prime}(0)$ is the dimensionless temperature gradient at sheet and $\operatorname{Re}_{x}=\frac{\rho c x^{2}}{\mu}$ is the local Reynolds number. It is obvious that the flow is influenced by the ferromagnetic parameter $\beta$. It is more fascinating and suitable to replace the dimensionless wall heat transfer rate $-\theta_{1}^{\prime}=-\left[\theta_{1}^{\prime}(0)+\xi^{2} \theta_{2}^{\prime}(0)\right]$ by the dimensionless and independent of the distance $\xi$ ratio $\theta^{*}(0)=\frac{\theta_{1}^{\prime}(0)}{\theta_{1}^{\prime}(0) \mid \beta=0}$ called heat transfer rate at the surface.

\section{METHODOLOGY}

Equations (18) to (22), (24) and (25) subject to boundary conditions (23) and (26) are highly non-linear, which are solved numerically by efficient Runge-Kutta method based on shooting technique. The higher-order differential equations are first reduced into a set of first order ODEs and then integrated as an initial value (IVP). 
The step-size is taken as $\nabla \eta=0.01$ trial values of $f^{\prime \prime}(0)$, $\theta_{1}^{\prime}(0), \theta_{2}^{\prime}(0), F(0)$ and $\theta_{p}(0)$ and were set iteratively by Newton-Raphson's method in order to satisfy the far field boundary condition asymptotically, i.e. $\eta \rightarrow \infty$ is satisfied at a finite value (take $\eta=10$ ).

\section{RESULTS AND DISCUSSION}

The effects of various pertinent parameters, namely, the ferromagnetic parameter $\beta$, Prandtl number $\operatorname{Pr}$, heat source/sink parameter $H_{1}$ and Eckert number $E c$ in the flow and heat transfer of ferromagnetic and dusty fluid were investigated with the help of figures and tables. The default values of the parameters for computational work were taken as $\operatorname{Pr}=0.72, \lambda=0.01, \varepsilon=2.0, \alpha_{1}=1.0$, $l_{1}=1, \quad c_{1}=c_{2}=c_{3}=1, \quad E=1, \quad N=0.5, \quad \alpha=0.2, \quad G^{*}=0.1$. To validate the accuracy of the present study, the value of $\theta_{1}^{\prime}(0)$ for different values of $P r$ are given in Table 1. The present numerical results are found to be in good agreement with those of Pal and Mondal (2010), and Roopa et al. (2011). Further Table 2 displays the values of skin-friction coefficient and the local Nusselt number for two types of heating processes, namely, PST and PHF for different pertinent parameters.
Table 1: Comparison of local Nusselt number $-\theta_{1}^{\prime}(0)$ for various values of $\operatorname{Pr}$ with $\alpha=N=H_{1}=\beta=E_{c}=G^{*}=0$

\begin{tabular}{lccc}
\hline$P r$ & $\begin{array}{c}\text { Pal and Mondal } \\
(2010)\end{array}$ & $\begin{array}{c}\text { Roopa } \text { et al. } \\
(2011)\end{array}$ & Present work \\
\hline & & & \\
\hline 0.72 & ----- & 1.08855 & 1.08863 \\
1 & 1.33333 & 1.33333 & 1.33334 \\
2 & 1.99999 & 1.99999 & 1.99999 \\
3 & 2.50971 & 2.50971 & 2.50967 \\
4 & 2.93878 & 2.93878 & 2.93873 \\
5 & 3.31647 & 3.31648 & 3.31645 \\
6 & 3.65776 & 3.65777 & 3.65773 \\
7 & 3.97150 & 3.97151 & 3.97145 \\
8 & 4.26345 & 4.26345 & 4.26337 \\
9 & 4.53760 & 4.53760 & 4.53749 \\
10 & 4.79687 & 4.79687 & 4.79671 \\
\hline
\end{tabular}

The temperature distribution of ferrofluid $\theta_{1}(\eta)$ and dust phase $\theta_{p}(\eta)$ for various values of the ferromagnetic interaction parameter $\beta$ for two cases PST and PHF are illustrated graphically in Figures 2(a) and 2(b). The figure shows that the temperature increases for ferrofluid and dust phase as $\beta$ increases in both PST and

Table 2: Skin-friction coefficient and Nusselt number for several values of physical parameter $\operatorname{Pr}=0.72$, $E c=2.0, \beta=0.2, H_{1}=0.2, N=0.5, \alpha=0.2$

\begin{tabular}{ccccccccc}
\hline$\alpha$ & $\beta$ & $H_{I}$ & $E c$ & $N$ & $P r$ & $-f^{\prime \prime}(0)$ & $\theta_{I}^{\prime}(0) \mathrm{PST}$ & $1 / \theta_{1}(0) \mathrm{PHF}$ \\
\hline 0.2 & 0.2 & 0.2 & 2.0 & 0.5 & 0.72 & 1.1591 & -0.2921 & 0.7181 \\
0.4 & & & & & & 1.2145 & -0.2841 & 0.7151 \\
0.6 & & & & & & 1.2566 & -0.2732 & 0.7110 \\
0.2 & 0.2 & 0.2 & 2.0 & 0.5 & 0.72 & 1.1591 & -0.2921 & 0.7181 \\
& 1.8 & & & & & 1.3990 & -0.1709 & 0.6531 \\
& 1.5 & & & & & 1.6924 & -0.0159 & 0.5271 \\
0.2 & 0.2 & -0.2 & 2.0 & 0.5 & 0.72 & 1.1553 & -0.5230 & 0.5929 \\
& & 0 & & & & 1.1569 & -0.4209 & 0.6157 \\
& & 0.2 & & & & 1.1591 & -0.2921 & 0.7181 \\
0.2 & 0.2 & 0.2 & 0.5 & 0.5 & 0.72 & 1.1524 & -0.8773 & 0.8957 \\
& & & 1.5 & & & 1.1568 & -0.4878 & 0.6697 \\
& & & 2.0 & & & 1.1591 & -0.2921 & 0.5929 \\
0.2 & 0.2 & 0.2 & 2.0 & 0.5 & 0.72 & 1.1591 & -0.2921 & 0.7181 \\
& & & & 1.0 & & 1.1585 & -0.2676 & 0.7142 \\
& & & & 1.5 & & 1.1582 & -0.2358 & 0.7106 \\
0.2 & 0.2 & 0.2 & 2.0 & 0.5 & 0.72 & 1.1591 & -0.2921 & 0.7181 \\
& & & & & 1.0 & 1.1575 & -0.3224 & 0.7770 \\
& & & & & 1.5 & 1.1558 & -0.3317 & 0.8343 \\
\hline
\end{tabular}




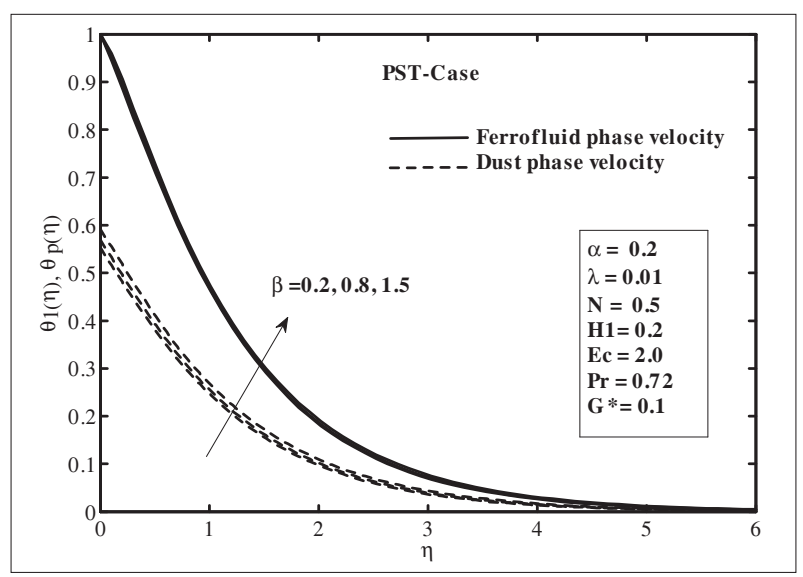

(a)

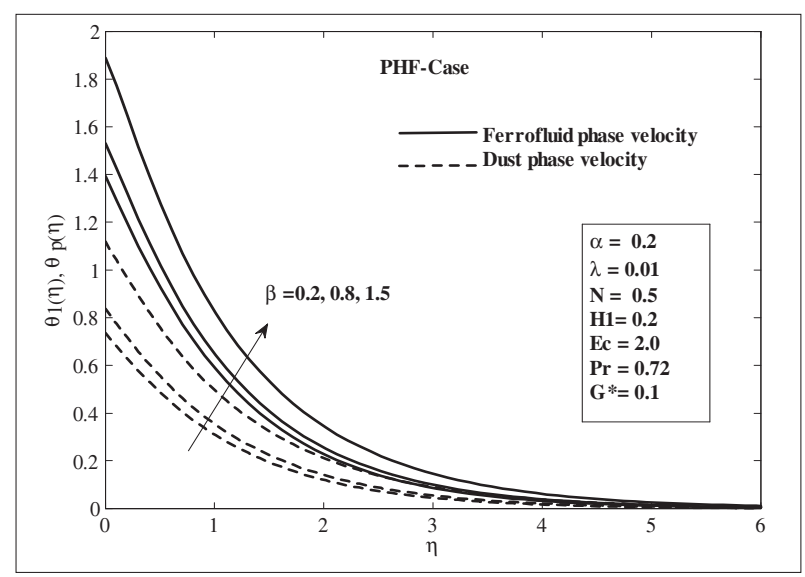

(b)

Figure 2: Influence of ferromagnetic parameter $(\beta)$ on temperature versus $\eta$ : for both (a) PST and (b) PHF cases

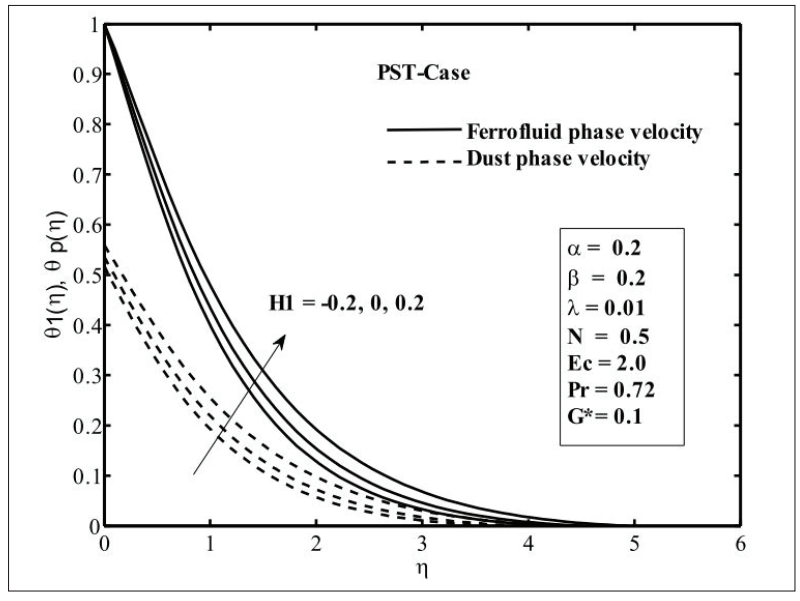

(a)

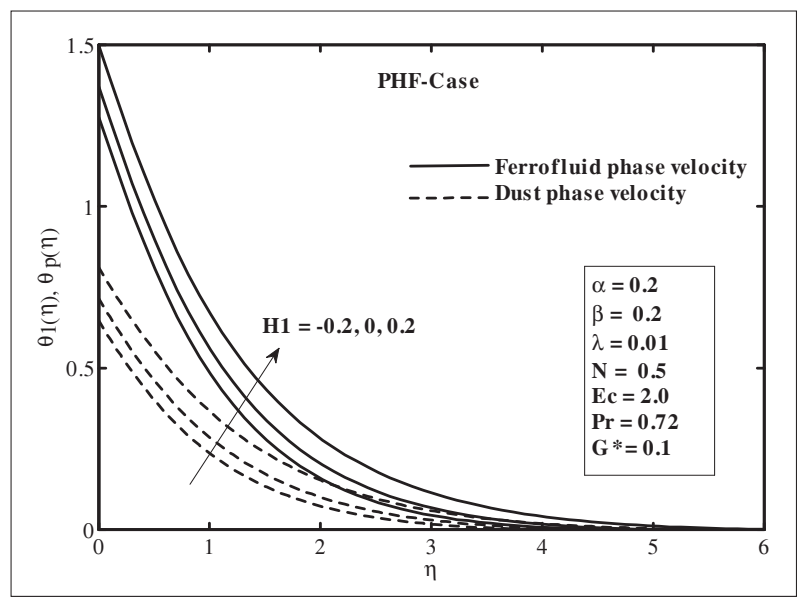

(b)

Figure 3: Influence of heat source/sink $\left(H_{1}\right)$ on temperature versus $\eta$ : for both (a) PST and (b) PHF cases

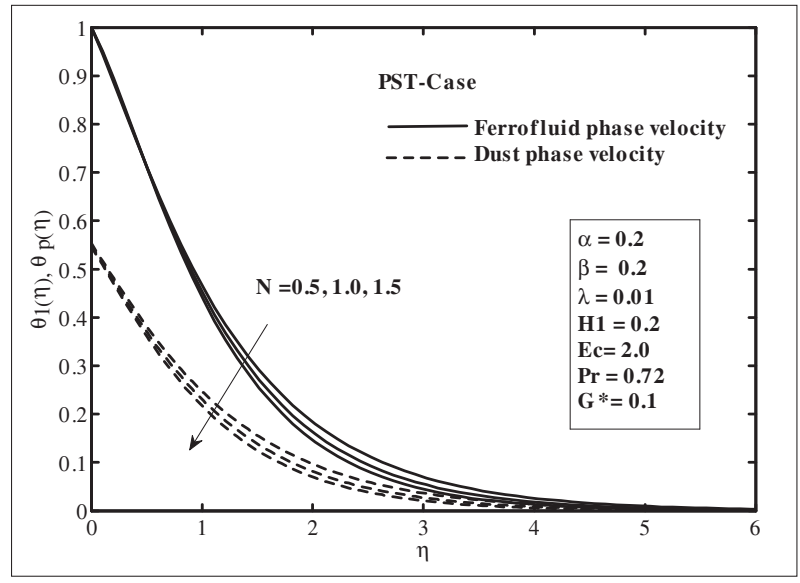

(a)

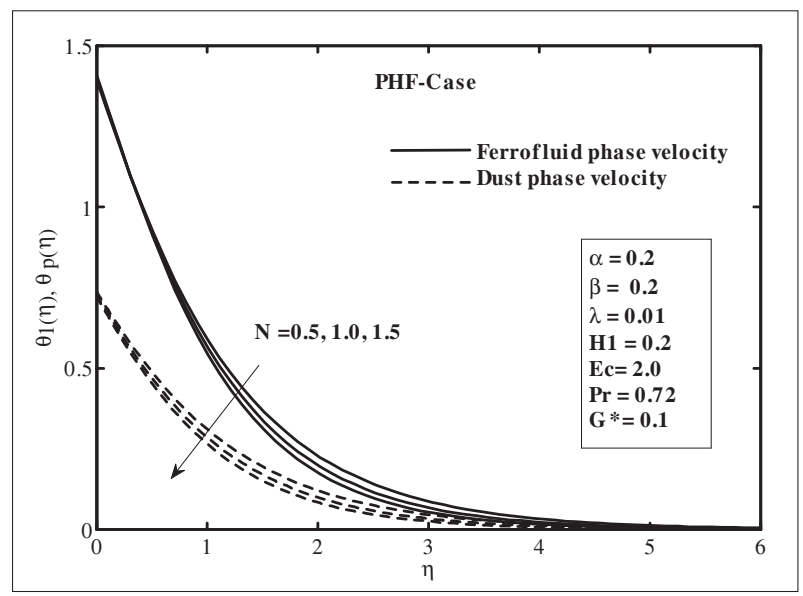

(b)

Figure 4: Influence of density of the dusty particle on temperature versus $\eta$ : for both (a) PST and (b) PHF cases 
PHF cases. It was also noticed that the ferromagnetic interaction parameter $\beta$ demonstrates the influence of applied magnetic field produced by a magnetic dipole on the fluid dynamics. The existence of an applied magnetic field act as the delaying force on the velocity profile, then as $\beta$ increases, so does the delaying force. Hereafter the axial velocity decreases whereas the reverse trend is exhibited for the temperature profile as seen in Figure 2. It was observed that the temperature of the ferrofluid is higher than the dust phase.

Figures 3(a) and 3(b) depict the temperature distribution $\theta_{1}(\eta)$ and $\theta_{p}(\eta)$ for different values of heat source/sink parameter $H_{1}$. It is concluded that the thermal boundary generates the energy, and this results

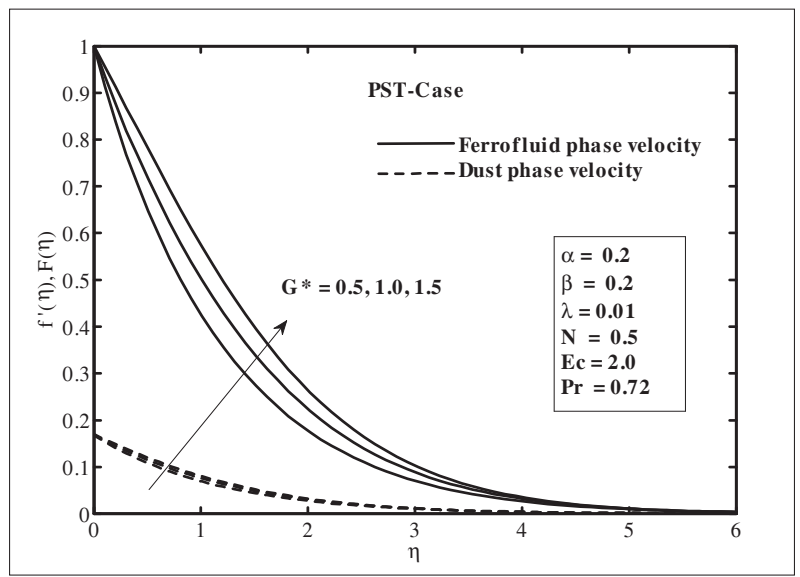

(a)

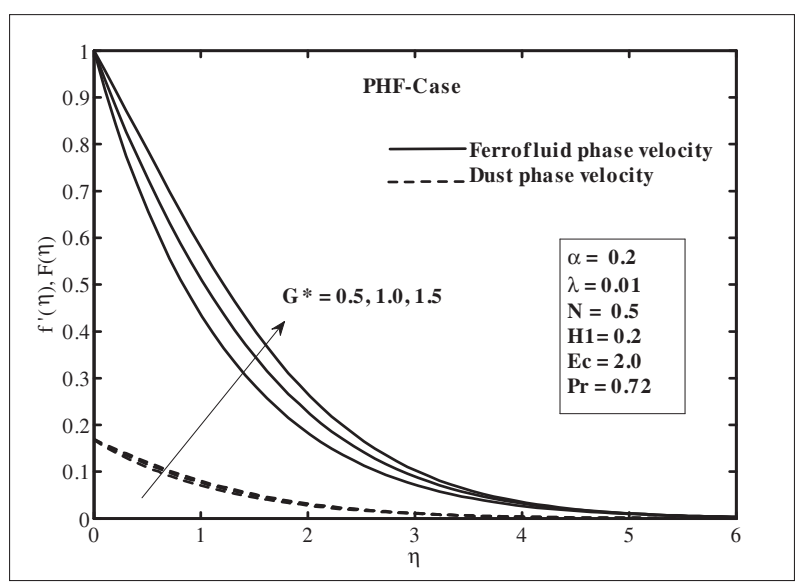

(b)

Figure 5: Influence of mixed convection parameter $\left(G^{*}\right)$ on velocity versus $\eta$ : for both (a) PST and (b) PHF cases the temperature to increase with an increase in the heat source $\left(H_{1}>0\right)$ parameter for PST and PHF, whereas the temperature decreases in the case of heat sink $\left(H_{1}<0\right)$.

Figures 4(a) and 4(b) show the influence of number density $(N)$ on temperature distribution $\theta_{1}(\eta)$ and $\theta_{p}(\eta)$ for PST and PHF. By analysing the graph, it is obvious that the temperature of ferrofluid and dust particles decreases with increasing the value of $N$. Also notice that the appearance of ferromagnetic particles is parallel to dust particles for both cases.

Figures 5(a) and 5(b) represent the impact of mixed convection parameter $G^{*}$ on velocity profiles $\mathrm{f}^{\prime}(\eta)$ and $\mathrm{F}(\eta)$ for PST and PHF cases. The velocity of ferrofluid

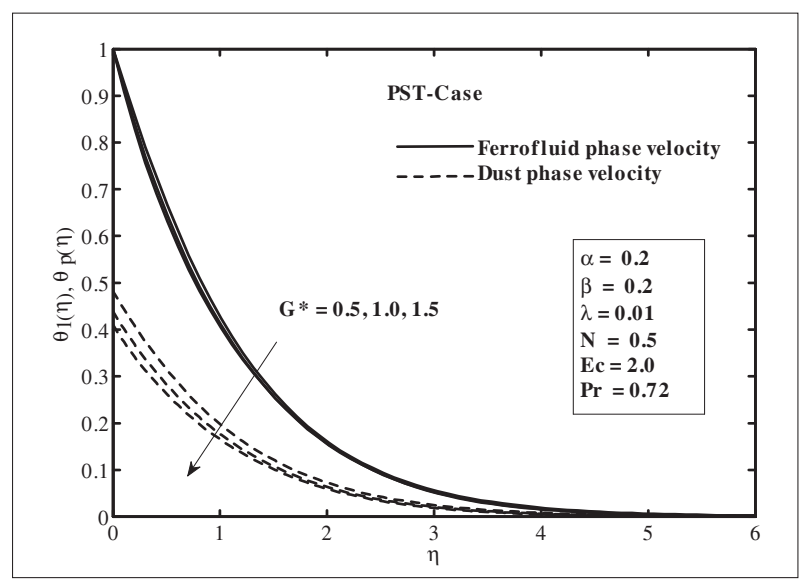

(a)

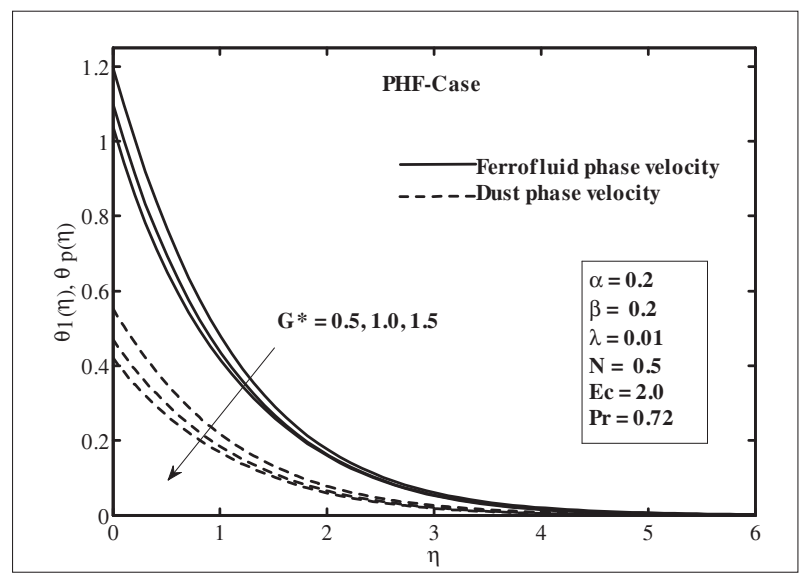

(b)
Figure 6: Influence of mixed convection parameter $\left(G^{*}\right)$ on temperature versus $\eta$ : for both (a) PST and (b) PHF cases 
and dust phase increases with increasing the value of mixed convection parameter $G^{*}$. The behaviour of buoyancy force is to accelerate the fluid within the boundary layer like a pressure gradient, whereas the effect of $G^{*}$ is to decrease the temperature profile within the boundary layer as pointed out in Figures 6(a) and $6(b)$.

Figures 7(a) and 7(b) indicate the effect of Eckert number $(E c)$ on temperature distribution $\theta_{1}(\eta)$ and $\theta_{p}(\eta)$ for both PST and PHF cases. From the graph it is observed that the temperature distributions for ferro and dusty fluid increase by increasing the value of $E c$ for both cases, as the heat energy is stored in the fluid due to frictional heating. By increasing $E c$ the temperature will enhance at any point and it is true in both cases.

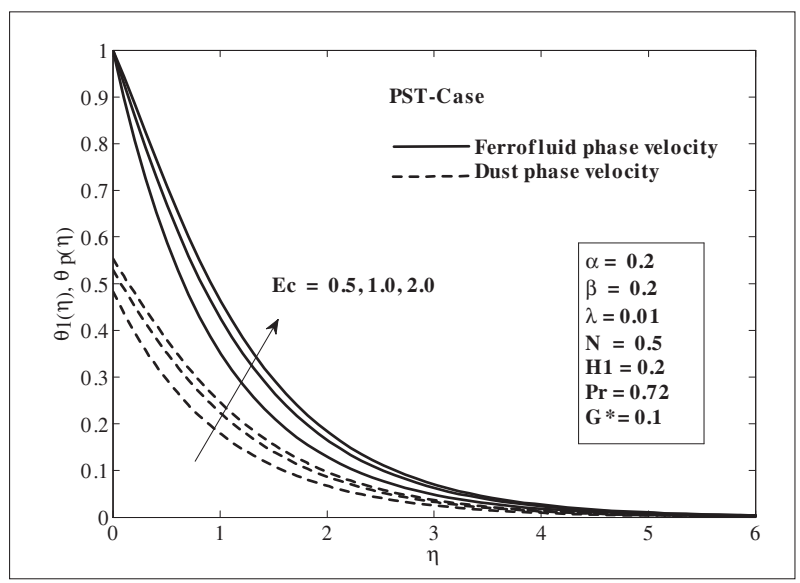

(a)

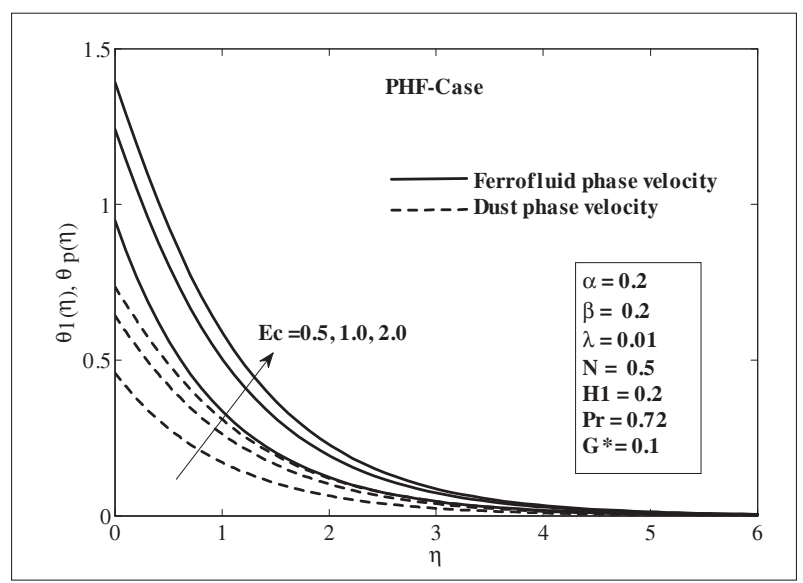

(b)

Figure 7: Influence of Eckert number $(E c)$ on temperature versus $\eta$ : for both (a) PST and (b) PHF cases

\section{CONCLUSION}

In the present article, numerical analysis is performed to examine the heat transfer of ferromagnetic and dusty fluid flow past a stretching surface in the presence of magnetic dipole with viscous dissipation and heat source/sink. The influence of secondary parameters such as Eckert number $(E c)$, Prandtl number $(P r)$, density of dust particles $(N)$ and ferromagnetic interaction parameter $(\beta)$ on velocity and temperature profiles are discussed in detail. The present numerical results agreed very well with those available in literature when $\alpha=N=H_{1}=\beta=E_{c}=G^{*}=0$. Some of the key points from the present work are: temperature profiles increase for both ferrofluid and dust phase with increasing the value of $\beta, H_{1}$ and $E c$; temperature distribution decreases for both ferrofluid and dust phases with increasing the values of $\operatorname{Pr}$ and $N$; the values of local Nusselt number show an increasing effect for PST and PHF with the variation of $H_{1}$.

\section{REFERENCES}

Akbar N.S., Nadeem S., Haq R.U. \& Khan Z.H. (2013). Numerical solutions of magnetohydrodynamic boundary layer flow of tangent hyperbolic fluid towards a stretching sheet. Indian Journal of Physics 87(11): 1121 - 1124. DOI: https://doi.org/10.1007/s12648-013-0339-8

Aminfar H., Mohammadpourfard M. \& Narrimanikahnamouei Y. (2011). A 3D numerical simulation of mixed convection of a magnetic nanofluid in the presence of non-uniform magnetic field in a vertical tube using two phase mixture model. Journal of Magnetism and Magnetic Materials 32: $1963-1972$.

DOI: https://doi.org/10.1016/j.jmmm.2011.02.039

Andersson H.I. \& Valnes O.A. (1998). Flow of a heated ferrofluid over a stretching sheet in the presence of a magnetic dipole. Acta Mechanica 128: 39 - 47. DOI: https://doi.org/10.1007/BF01463158

Chang C.H., Tan C.W., Miao J. \& Barbastathis G. (2009). Self-assembled ferrofluid lithography: patterning micro and nanostructures by controlling magnetic nanoparticles. Nanotechnology 20(49): 495301. DOI: https://doi.org/10.1088/0957-4484/20/49/495301

Feynman R.P., Leighton R.B. \& Sands M. (1963). The Feynman Lectures on Physics. Addison-Wesley, Reading, USA.

Freidoonimehr N., Rashidi M.M. \& Mahmud S. (2015). Unsteady MHD free convective flow past a permeable stretching vertical surface in a nanofluid. International Journal of Thermal Science 87: 136 - 145. DOI: https://doi.org/10.1016/j.ijthermalsci.2014.08.009

Ganguly R., Sen S. \& Puri I.K. (2004). Heat transfer augmentation using a magnetic fluid under the influence of a line dipole. Journal of Magnetism and Magnetic Materials 271: 63 - 73 . 
DOI: https://doi.org/10.1016/j.jmmm.2003.09.015

Ghasemian M., Najafian Z., Ashrafi M. \& Goharkhah A.M. (2015). Heat transfer characteristics of $\mathrm{Fe}_{3} \mathrm{O}_{4}$ ferrofluid flowing in a mini channel under constant and alternating magnetic fields. Journal of Magnetism and Magnetic Materials 381: $158-167$.

DOI: https://doi.org/10.1016/j.jmmm.2014.12.078

Gireesha B.J., Chamkha A.J., Rudraswamy N.G. \& Krishnamurthy M.R. (2015). MHD flow and heat transfer of a nanofluid embedded with dust particles over a stretching sheet. Journal of Nanofluids 4(1): $66-72$.

DOI: https://doi.org/10.1166/jon.2015.1126

Gireesha B.J., Mahanthesh B. \& Gorla R.S.R. (2014). Suspended particle effect on nanofluid boundary layer flow past a stretching surface. Journal of Nanofluids 3(3): $267-277$.

DOI: https://doi.org/10.1166/jon.2014.1101

Gireesha B.J., Ramesh G.K., Abel M.S. \& Bagewadi C.S. (2011). Boundary layer flow and heat transfer of a dusty fluid flow over a stretching sheet with non-uniform heat source/sink. International Journal of Multiphase Flow 37(8): $977-982$.

Kefayati G. (2014). Natural convection of ferrofluid in a linearly heated cavity utilizing LBM. Journal of Molecular Liquids 19: 1 - 9 .

DOI: https://doi.org/10.1016/j.molliq.2013.11.021

Majeed A., Zeeshan A. \& Ellahi R. (2016a). Unsteady ferromagnetic liquid flow and heat transfer analysis over a stretching sheet with the effect of dipole and prescribed heat flux. Journal of Molecular Liquids 223: 528 - 533. DOI: https://doi.org/10.1016/j.molliq.2016.07.145

Majeed A., Zeeshan A., Rashidi M.M. \& Arain M.B. (2016b). Stagnation point flow of ferromagnetic particle-fluid suspension over a stretching/shrinking surface in a porous medium with heat source/sink. Caspian Journal of Applied Sciences Research 5(3): $34-44$.

Nandkeolyar R., Seth G.S., Makinde O.D., Sibanda P. \& Ansari M.S. (2013). Unsteady hydromagnetic natural convection flow of a dusty fluid past an impulsively moving vertical plate with ramped temperature in the presence of thermal radiation. Journal of Applied Mechanics 80(6): 061003. DOI: https://doi.org/10.1115/1.4023959

Nawaz M. \& Hayat T. (2014). Axisymmetric stagnation-point flow of nanofluid over a stretching surface. Advances in Applied Mathematics and Mechanics 6(2): 220 - 232. DOI: https://doi.org/10.4208/aamm.2013.m93

Nawaz M., Hayat T. \& Alsaedi A. (2012). Dufour and soret effects on MHD flow of viscous fluid between radially stretching sheets in porous medium. Applied Mathematics and Mechanics 33(11): 1403 - 1418.

DOI: https://doi.org/10.1007/s10483-012-1632-6

Pal D. \& Mondal H. (2010). Hydromagnetic non-Darcy flow and heat transfer over a stretching sheet in the presence of thermal radiation and ohmic dissipation. Communications in Nonlinear Science and Numerical Simulation 15(5): $1197-1209$.

DOI: https://doi.org/10.1016/j.cnsns.2009.05.051

Rashidi M.M., Freidoonimehr N., Hosseini A., Bég O.A. \&
Hung T.K. (2014a). Homotopy simulation of nanofluid dynamics from a non-linearly stretching isothermal permeable sheet with transpiration. Meccanica 49(2): $469-482$.

DOI: https://doi.org/10.1007/s11012-013-9805-9

Rashidi M.M., Ganesh N.V., Hakeem A.A. \& Ganga B. (2014b). Buoyancy effect on MHD flow of nanofluid over a stretching sheet in the presence of thermal radiation. Journal of Molecular Liquids 198: 234 - 238.

DOI: https://doi.org/10.1016/j.molliq.2014.06.037

Rehman S.U., Zeeshan A., Majeed A. \& Arain M.B. (2017). Impact of Cattaneo-Christov heat flux model on the flow of Maxwell ferromagnetic liquid along a cold flat plate embedded with two equal magnetic dipoles. Journal of Magnetics 22(3): $472-477$.

DOI: https://doi.org/10.4283/JMAG.2017.22.3.472

Roopa G.S., Gireesha B.J. \& Bagewadi C.S. (2011). Effect of viscous dissipation on MHD flow and heat transfer of a dusty fluid over an unsteady stretching sheet. International Journal of Mathematical Archive 2(11): 2229 - 5046.

Rosensweig R.E., Nestor J.W. \& Timmins R.S. (1965). Ferrohydrodynamic Fluids for Direct Conversion of Heat Energy. Institution of Chemical Engineers, London, UK.

Saffman P.G. (1962). On the stability of laminar flow of a dusty gas. Journal of Fluid Mechanics 13(1): 120 - 128. DOI: https://doi.org/10.1017/S0022112062000555

Shehzad N., Zeeshan A., Ellahi R. \& Vafai K. (2016). Convective heat transfer of nanofluid in a wavy channel: Buongiorno's mathematical model. Journal of Molecular Liquids 222: 446 - 455.

DOI: https://doi.org/10.1016/j.molliq.2016.07.052

Sheikholeslami M. \& Ganji D.D. (2014). Ferrohydrodynamic and magnetohydrodynamic effects on ferrofluid flow and convective heat transfer. Energy 75: $400-410$.

DOI: https://doi.org/10.1016/j.energy.2014.07.089

Sheikholeslami M. \& Rashidi M.M. (2015). Effect of space dependent magnetic field on free convection of $\mathrm{Fe}_{3} \mathrm{O}_{4}-$ water nanofluid. Journal of Taiwan Institute of Chemical Engineering 56: 6 - 15.

DOI: https://doi.org/10.1016/j.jtice.2015.03.035

Sheikholeslami M., Rashidi M.M. \& Ganji D.D. (2015). Effect of non-uniform magnetic field on forced convection heat transfer of $\mathrm{Fe}_{3} \mathrm{O}_{4}$-water nanofluid. Computer Methods Application of Mechanical Engineering 294: 299 - 312.

DOI: https://doi.org/10.1016/j.cma.2015.06.010

Shliomis M.I. (2004). Ferrofuids as thermal ratchets. Physical Review Letters 92(18): 188901.

DOI: https://doi.org/10.1103/PhysRevLett.92.188901

Song W.B., Kim H., Son C. \& Ziaie B. (2006). Fabrication of polymeric 3-D micro-structures using ferrofluid molds, $19^{\text {th }}$ IEEE International Conference on Micro Electro Mechanical Systems, 22 - 26 January, Istanbul, Turkey, pp. $334-337$.

Strek T. \& Jopek H. (2007) Computer simulation of heat transfer through a ferrofluid. Physica Status Solidi (b) 244(3): $1027-1037$.

DOI: https://doi.org/10.1002/pssb.200572720 
Zeeshan A. \& Majeed A. (2016). Effect of magnetic dipole on radiative non-Darcian mixed convective flow over a stretching sheet in porous medium. Journal of Nanofluids 5(4): $617-626$.

DOI: https://doi.org/10.1166/jon.2016.1237

Zeeshan A., Majeed A. \& Ellahi R. (2016). Effect of magnetic dipole on viscous ferro-fluid past a stretching surface with thermal radiation. Journal of Molecular Liquids 215:
$549-554$.

DOI: https://doi.org/10.1016/j.molliq.2015.12.110

Zeeshan A., Majeed A., Fetecau C. \& Muhammad S. (2017). Effects on heat transfer of multiphase magnetic fluid due to circular magnetic field over a stretching surface with heat source/sink and thermal radiation. Results in Physics 7: $3353-3360$.

DOI: https://doi.org/10.1016/j.rinp.2017.08.047 